\title{
AUTOMATED PROCESSING OF POINT CLOUDS TO UPDATE LAND REGISTRY MAPS
}

\author{
Bence Péter Hrutka ${ }^{1}$, Zoltán Siki ${ }^{1}$, Bence Takács ${ }^{1}$ \\ ${ }^{1}$ Department of Geodesy and Surveying, Budapest University of Technology and Economics, Hungary \\ Email: hrutka.bence@edu.bme.hu, siki.zoltan@emk.bme.hu, takacs.bence@emk.bme.hu
}

KEY WORDS: Land registry map, geometric quality, point cloud techniques, classification, segmentation, surface normal, region growing, RANSAC, Normalised Digital Surface Model, edge detection.

\begin{abstract}
:
The quality control, maintenance, and renewal of land registry maps have always been priorities in the surveying profession. Many countries worldwide must face the issue that a significant part of their current digital land registry maps are based on old analogue maps that were digitised without involving any in-situ measurements. A direct consequence of this is that the digitised maps' accuracy leaves much to be desired and lags behind maps based on either correct survey or numerical data. Moreover, the quality of existing digital maps can be characterised by inhomogeneity that highly depends on the location. The final solution to the problem would be to carry out new surveys in the critical areas, but that has been postponed due to the lack of time and excessive costs.

However, in recent years, point cloud technologies, such as Unmanned Aerial Vehicles (UAV), Terrestrial Laser Scanners (TLS), Aerial Laser Scanners (ALS), together with Mobile Mapping Systems (MMS), have become the focus of attention in mapping. Thanks to these technologies, experts can survey large areas with the necessary and homogenous accuracy, high resolution, and significantly, very rapidly. It is beyond doubt that these modern technologies benefit the process of updating old and less relevant maps.

Another underlying aspect worth considering is the automation in data processing since a massive amount of data needs to be evaluated. Some algorithms and their validation on study areas in Hungary are presented in this paper. Our study focuses on the mapping of buildings using point clouds generated from UAV images.
\end{abstract}

\section{INTRODUCTION}

Updating the land registry map using state-of-the-art land surveying methods has gradually become the focus of attention over the past few decades. In many countries, the original land registry maps were developed as analogue maps, frequently back in the 19th century. The primary issue is that the original maps were manually redrawn several times over the past century. Finally, some time around the beginning of the $21 \mathrm{st}$ century, these maps were digitised. Each redraw, as well as digitalisation, aggravated their geometric inaccuracy. It is quite common to have a few meters offset in the features depicted in the land registry maps, which yields a wide variety of problems in applying maps, such as in public utility registration and engineering practice, like planning. It can be stated with complete confidence that many countries worldwide are facing the same issue (Hanus et al., 2017; Ceh et al., 2019).

At the same time, first-rate surveying techniques, especially Unmanned Aerial Vehicles (UAV) can provide users with a fast and affordable solution. Point clouds derived from digital images taken from UAV were applied in the Netherlands to identify property boundaries (Rijsdisk et al., 2013). It was concluded that the required accuracy is achievable without entering the properties, which can significantly alleviate the work in the field. Accuracy of the final product was checked by GNSS measurements, and it was proved to be below $10 \mathrm{~cm}$. Similar tests were completed in Albania (Barnes et al., 2014) and Poland (Kedzierski et al., 2016; Kurczynski et al., 2016), in both countries, there was a high demand to update the old land registry maps.
Another application worth considering is the survey of disaster areas. Considerable deviations can be attributed to severe landslides; consequently, extended areas need to be surveyed quickly, as is presented in (Gutierrezze et al., 2019). Although pure photogrammetry can provide the required accuracy, UAV photogrammetry can be extended by LIDAR technology to increase efficiency and accuracy ( $\mathrm{He}$ and $\mathrm{Li}, 2020)$.

The discussion of this topic would be incomplete without mentioning the role of open-source software widely used in this field. OpenDroneMap (ODM) offers a terrific opportunity to post-process aerial images and generate point clouds either in a web-based interface (WebODM) or in a command line (OpenDronMap Development Team, 2021). CloudCompare can be considered a general open-source tool for a wide variety of manipulations on point clouds, including the original objective, such as comparing different point clouds or meshes (CloudCompare Development Team, 2021). QGIS (Cutts and Graser, 2018) and GRASS GIS (Neteler and Mitasova, 2008) are widely used to create, edit, visualise, analyse, and publish geospatial information, including meshes and point clouds. This open-source software has Graphical User Interface (GUI) and Command Line Interface (CLI), which can be programmed for automation using Python as the overall programming language. In addition to the abovementioned software, there are a series of open-source Python libraries, like Scikit-learn, SciPy, and Open3D (Zhou et al., 2018), to support automatic data processing of point clouds. 


\section{MATERIALS AND METHODS}

\subsection{Segmentation and classification of buildings}

In the investigation of automated point cloud processing, significant reductions in file sizes are essential. In most processing software, like CloudCompare there are a wide variety of options such as resample. On the other hand, there is a risk of losing valuable points, which might cause difficulties in the later processing steps. Therefore, combining different segmentation and classifications methods can be effective (Nguyen and Le, 2013).

Pre-classification is unavoidable before the application of segmentation algorithms. To reduce the size of the point cloud, the CloudCompares' Cloth Simulation Filter (CSF) plugin (Zhang et al., 2016) can be the first option. With the help of this function, the points in the point cloud can be separated into two classes: ground points and non-ground points (Figure 1).

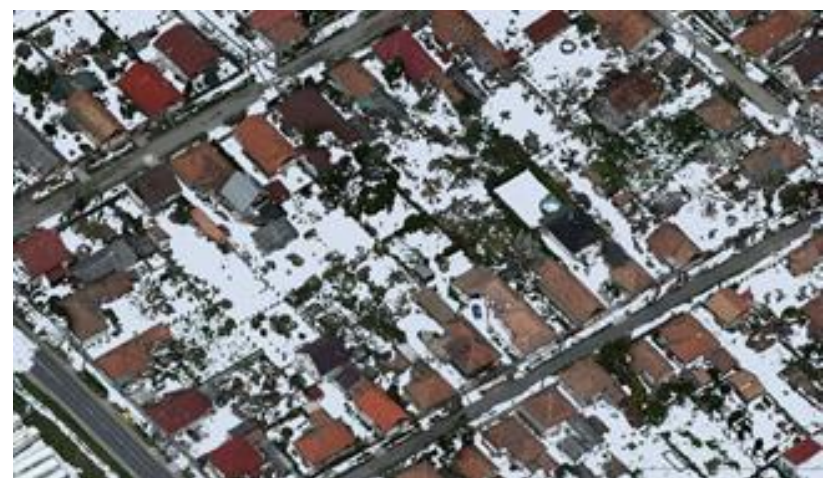

Figure 1. Cloth Simulation Filter (CSF) plugin's result, the nonground points.

Using the CSF's result, a Normalised Digital Surface Model (nDSM) can be created by computing the vertical distance between the non-ground points and a mesh generated from the ground points, then saving these distances as $\mathrm{Z}$ coordinates (Figure 2).

From the Normalized Digital Surface Model, different segmentation methods can be applied to separate points on the building walls. CloudCompare can compute the normal of the point cloud; consequently, elevations angle values can be derived at each point. Assuming that the walls of buildings are vertical, points with elevation angles close to $90^{\circ}$ can be filtered. Thanks to normalisation, another filter on $\mathrm{Z}$ coordinates, for instance between 0.3 and 2 meters, can be performed to cut low vegetation.

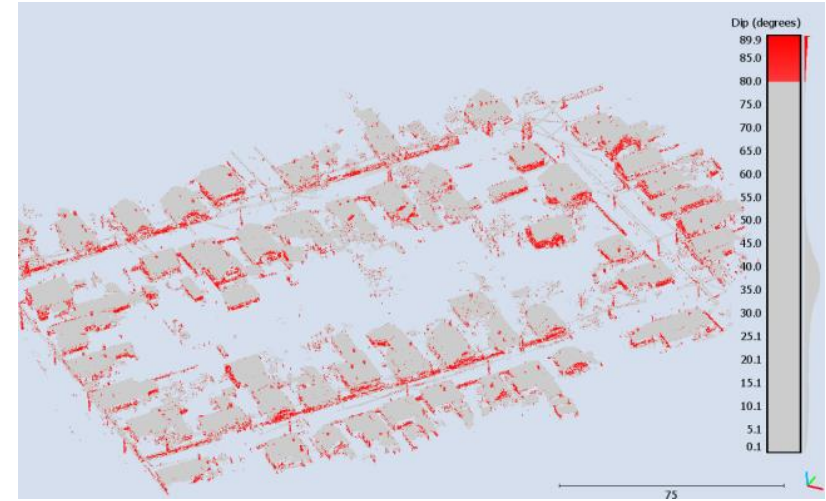

Figure 3. The filtered points with elevation angles between $80^{\circ}$ and $90^{\circ}$.

The result of the previous normal vector based segmentation method is usually not perfect. The remaining vegetation points cause noise. To mitigate this effect, a region growing algorithm can be applied. The normal vectors (Taylor, 2021) and the Gaussian curvature (Beksi, 2021) are calculated using neighbouring points within a certain radius (Murtiyoso, 2021). Knowing the normal directions and curvature values, the wall segments can be separated from each other. An examination of the normal directions and curvature values in a certain range within a given threshold can be used to obtain contiguous wall pieces.

The summary of the presented preparation steps is shown in the following flowchart (Figure 4).

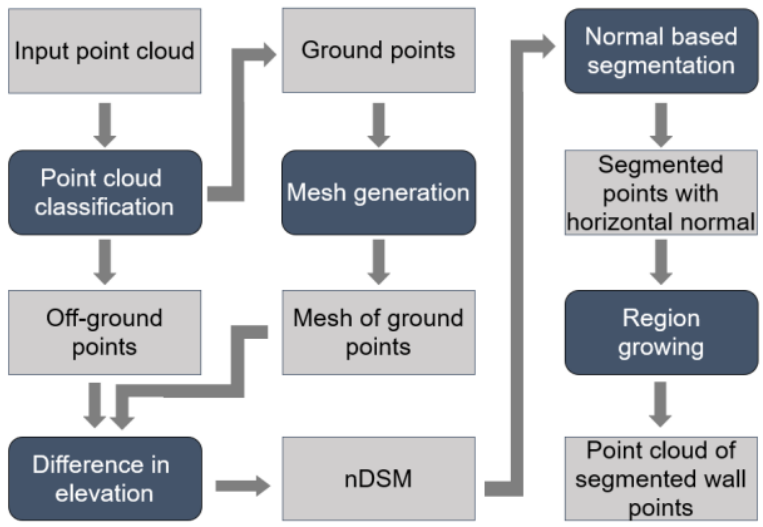

Figure 4. Flow chart of the point cloud pre-processing.
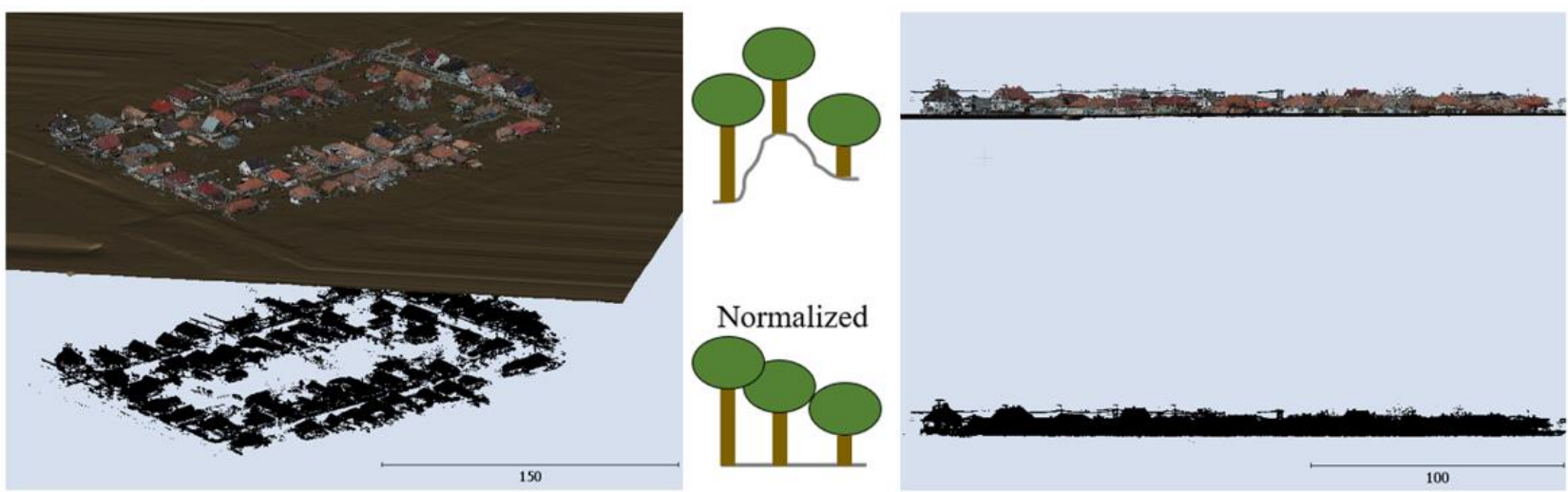

Figure 2. Process to generate normalised surface model from point cloud. 
To support automated building mapping, another simplification of leaving the $\mathrm{Z}$ coordinates is highly recommended, hence a $2 \mathrm{D}$ reduced point cloud is derived.

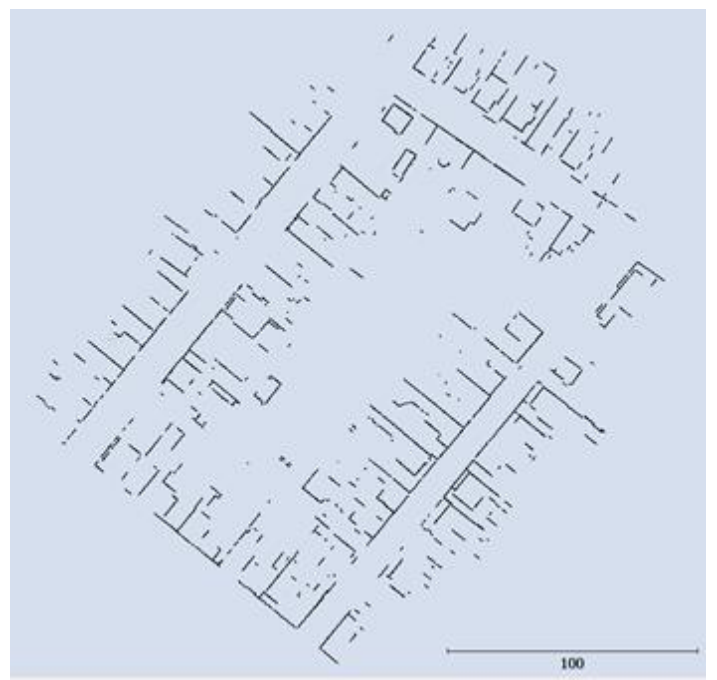

Figure 5. 2D reduced point cloud.

\subsection{Automated building detection}

As a result of classification and segmentation described in the previous chapter, we recommend three methods to map buildings using the $2 \mathrm{D}$ reduced point cloud: robust linear regression, sequential RANdom SAmple Consensus (RANSAC), and raster-vector conversion.

The simplest one of the three algorithms is based on robust linear regression using a Least Absolute Deviation method, which is widely known as L1-norm estimation. The results are the parameters of the detected lines and the coordinates of start and end points.

The second method is based on RANSAC (Fisher and Bolles, 1982). The principle of the method is that initial model parameters are determined using a minimal amount of data. In the next step, more points are involved to finalise the parameters, and only those are kept that fit within the threshold (Fouhey, 2011). Applying a Principal Component Analysis (PCA), the original point is worth splitting into smaller parts. Consequently, RANSAC can be applied sequentially to boost computation efficiency.

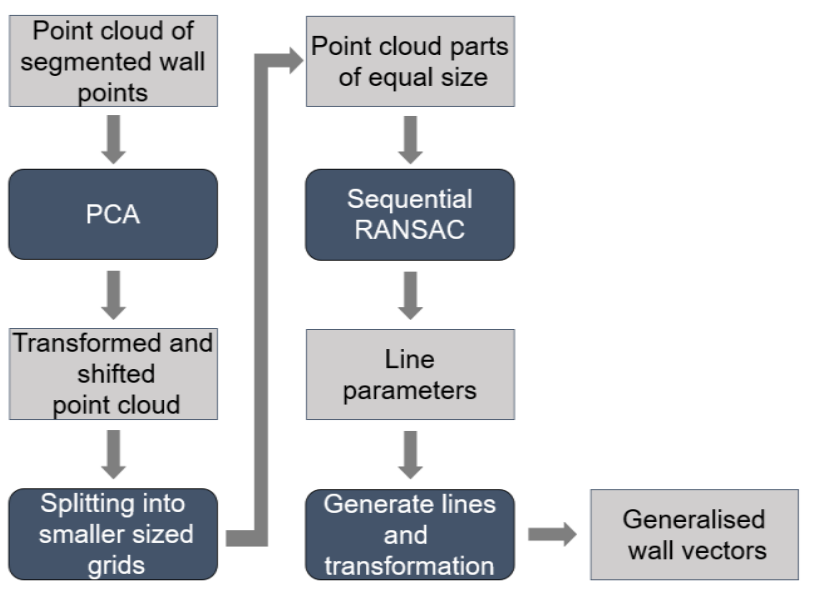

Figure 6. Flow chart of the sequential RANSAC based method.
The third method is slightly different from the previous two. From the segmented point cloud, a raster file with a specific geometric resolution needs to be created having pure black and white pixels.

Raster thinning might be useful before converting the black pixels back into vector lines. Ultimately, a Douglas-Peucker algorithm (Douglas et al., 1973) can be used for generalisation. The flowchart of the raster-vector conversion can be seen in Figure 7.

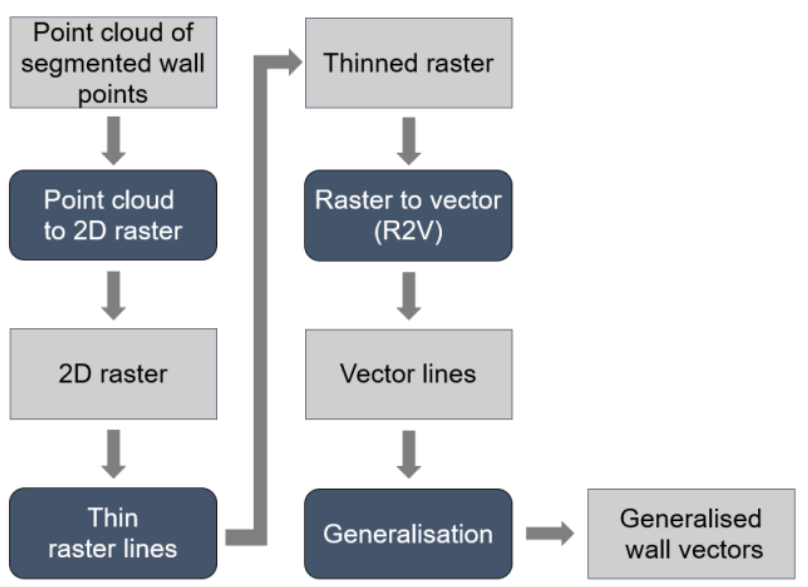

Figure 7. Flow chart of the raster-vector conversion.

GRASS's drawing clean-up tools can improve the results of all three methods.

\section{MEASUREMENTS AND IMAGE PROCESSING}

Two test areas were used during the evaluation of the methods. Photos were taken by a DJI Phantom 4 Pro UAV in autonomous missions on both test areas.

The first test area was Barnag, a small village in Hungary of approximately 38 hectares. There are more than 200 detached houses in the settlement. Nadir and oblique photos were taken at 55-meter altitude AGL (Above Ground Level), having a 20 MPixel camera with $1.5 \mathrm{~cm}$ GSD (Ground Sample Distance). The overlap among the adjacent images was $80 \%$, both in rows and between rows. A standard (zigzag) mission was planned for nadir and oblique photos; the oblique angle was 25 degrees from the vertical. Finally, 1400 nadir and 890 oblique photos were taken during the two missions. For precise georeferencing, GCPs (Ground Control Points) were used in both cases; their 3D positions were measured by RTK GNSS technology.

The second test area, a smaller, 3-hectare block in a small town near Budapest, called Üllö. Similar missions were completed, but the oblique images were made in a grid mission to increase the number of points derived on the walls of the buildings. These points are essential to find the footprint of buildings.

The photogrammetric processing was carried out mainly in ODM (OpenDroneMap) and WebODM, but we also tried 3DSurvey and Agisoft Metashape software. ODM and WebODM are some of the most popular open-source SFM (Structure from Motion) and MVS (Multi-view Stereo) programs. Four products were generated from the photos: (1) point cloud, (2) orthophoto, (3) DTM (Digital Terrain Model) and (4) DSM (Digital Surface Model). 


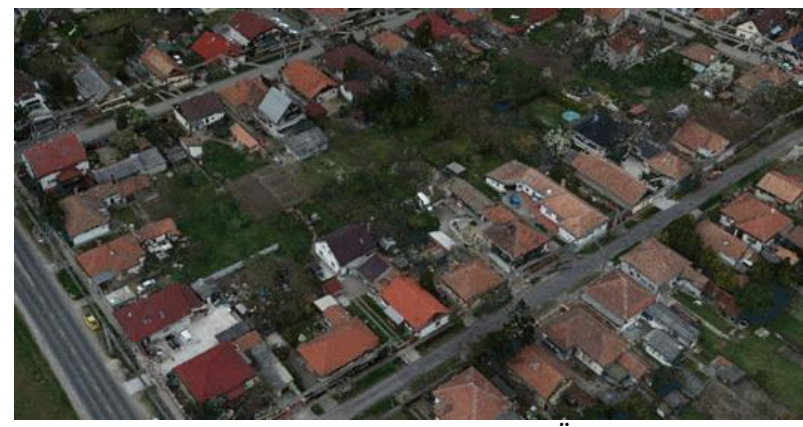

Figure 8. Dense point cloud of the Üllö test area.

For the quality control of the final products, some detail points were also measured combining RTK GNSS and total station observations.

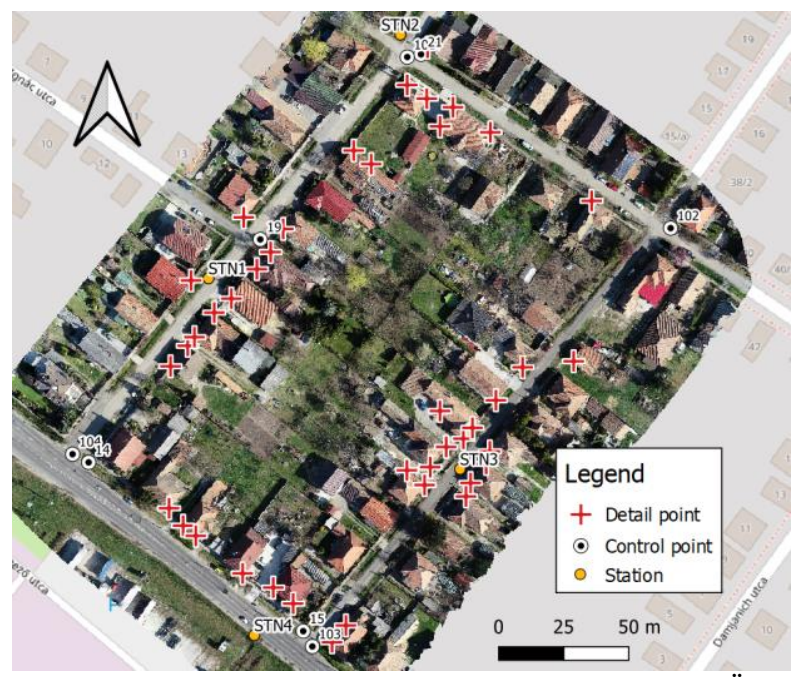

Figure 9. Ground control points and detail points in the Üllo test area.

\section{RESULTS}

The presented pre-process and automated building detection methods were performed on the point cloud of the smaller study area (Figure 10).

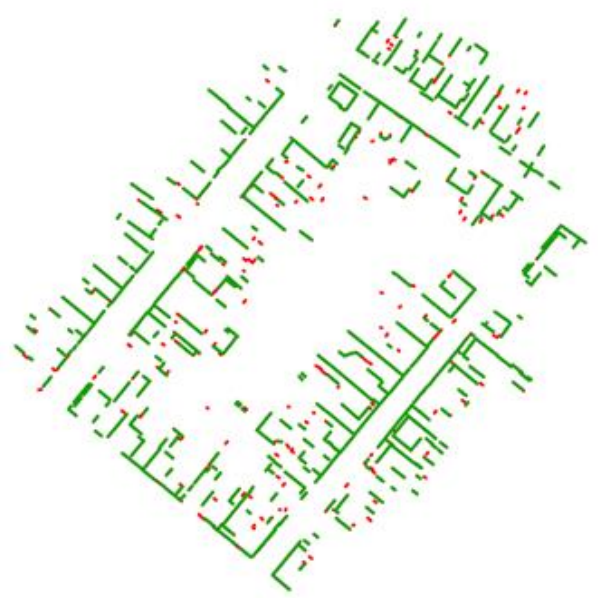

Figure 10. The cleaned result (green) of the robust linear regression.
The results of the tested methods are not perfect due to some original measurement issues. The underlying problem is the lack of points on certain walls, hidden by obstacles, especially trees.

Measured detail points were used to quantify and compare the accuracy of the three methods. Robust linear regression and raster-vector conversion methods proved to be more comprehensive since 35 out of the 41 corner and wall points (86\%) were found, while only 26 control points using the sequential RANSAC procedure.

In addition to completeness, accuracy was investigated by comparing the results of the three methods to the measured detail points (Table 1). There are only minor differences; however, linear regression seems to be the most accurate. The main accuracy parameters are considerably better than the accuracy of sometimes more than one-hundred-year-old analogue maps.

\begin{tabular}{|c|c|c|c|}
\hline \multicolumn{4}{|c|}{ Statistical measure of the results [cm] } \\
\hline & $\begin{array}{c}\text { Robust } \\
\text { linear } \\
\text { regression }\end{array}$ & $\begin{array}{c}\text { Sequential } \\
\text { RANSAC }\end{array}$ & $\begin{array}{c}\text { Raster- } \\
\text { vector } \\
\text { conversion }\end{array}$ \\
\hline Mean & 9 & 9 & 18 \\
\hline Minimum & 1 & 0 & 4 \\
\hline Maximum & 26 & 33 & 48 \\
\hline Median & 7 & 8 & 15 \\
\hline Std. dev. & 7 & 7 & 12 \\
\hline
\end{tabular}

Table 1. Statistical results of the Üllö test area.

On the larger Barnag test area, with the help of 36 measured points, the methods also were tested with similar results (Table 2).

\begin{tabular}{|c|c|c|c|}
\hline \multicolumn{4}{|c|}{ Statistical measure of the results [cm] } \\
\hline & $\begin{array}{c}\text { Robust } \\
\text { linear } \\
\text { regression }\end{array}$ & $\begin{array}{c}\text { Sequential } \\
\text { RANSAC }\end{array}$ & $\begin{array}{c}\text { Raster- } \\
\text { vector } \\
\text { conversion }\end{array}$ \\
\hline Mean & 12 & 12 & 19 \\
\hline Minimum & 0 & 1 & 3 \\
\hline Maximum & 41 & 36 & 69 \\
\hline Median & 11 & 11 & 16 \\
\hline Std. dev. & 9 & 8 & 13 \\
\hline
\end{tabular}

Table 2. Statistical results of the Barnag test area.

In addition to its accuracy, linear regression is the simplest method among the three analysed, since there is no need to set its parameters. While the underlying difficulty of applying a sequential RANSAC method is that it needs a series of parameters to set up, and their values could vary in space due to a wide variety of factors. Raster vector conversion is a wellknown method since it has been widely applied in cartography during the digitalization campaigns of analogue maps. It can yield a favourable resolution, but its accuracy slightly lags behind the two abovementioned methods. 


\section{CONCLUSION}

The main results of automated evaluation of point clouds derived from UAV photogrammetry have been presented in this article. The primary objective is to update land registry maps, focusing on building recognition and large-scale mapping. Photos taken from an oblique camera position are of utmost importance to get a relevant number of points on the walls of buildings.

The entire process, including image processing, derivation of a georeferenced point cloud, classification, the significance of getting 2D maps as a final product, can be completed using open-source software. In this paper, two methods for classification were analysed in detail: the first is based on filtering by surface normals, while the second on a region growing algorithm.

Upon successful separation of points on building walls, the automated mapping of buildings was analysed. Three methods have been investigated: first linear regression, secondly sequential RANSAC line fitting and finally a raster-vector conversion. All methods were validated by measurements using RTK GNSS and total station. Linear regression proved to be the most efficient and accurate method, characterised by $\pm 7 \mathrm{~cm}$ standard deviation. Another aspect worth considering is completeness, which was $86 \%$ in the case of linear regression. To further improve this result, manual evaluation, for example, to map the remaining part of buildings could be utilised.

\section{REFERENCES}

Barnes, G., Volkmann, W., Sherko, R., Kelm, K., 2014: Drones for Peace: Part 1 of 2. Design and Testing of a UAV-based Cadastral Surveying and Mapping Methodology in Albania. World Bank Conference On Land And Poverty, 2014. http://www.fao.org/fileadmin/user_upload/nr/land_tenure/UAS _paper_1_01.pdf (11 June 2021).

Beksi, W., 2021: Estimate Principal Curvatures. MATLAB Central File Exchange. Retrieved March 25, 2021. https://uk.mathworks.com/matlabcentral/fileexchange/46772estimate-principal-curvatures?s_tid=prof_contriblnk (11 June 2021).

CloudCompare Development Team, 2021. CloudCompare Software. Version 2.11.3 Anoia. https://www.danielgm.net/cc/ (11 June 2021).

Ceh, M., Gielsdorf, F., Trobec, B., Krivic, M., Lisec, A., 2019: Improving the Positional Accuracy of Traditional Cadastral Index Maps with Membrane Adjustment in Slovenia. International Journal of Geo-Information. July 2019. 8(8):338. doi:10.3390/ijgi8080338.

Cutts, A., Graser, A., 2018: Learn QGIS $4^{\text {th }}$ Edition. PacktPub ISBN 978-1-78899-742-3.

Douglas, D., Peucker, T., (1973): Algorithms for the reduction of the number of points required to represent a digitized line or its caricature, The Canadian Cartographer 10(2), 112-122 (1973) doi:10.3138/FM57-6770-U75U-7727.

Fischler, M.A., Bolles, R.C., 1981: Random sample consensus: A paradigm for model fitting with applications to image analysis and automated cartography. Communications of the ACM, 24(6), 381-395.
Fouhey, D.F., 2011: Multi-model estimation in the presence of outliers. PhD Thesis, Middlebury College.

Gutiérrez Viñuales, M., Puch Sleive, E., Chalabe, S., 2019: Experiences in cadastral restitution at a town affected by a natural disaster. The case of volcan, Jujuy Province, Argentina. The International Archives of the Photogrammetry, Remote Sensing and Spatial Information Sciences, Volume XLII2/W13, 2019 ISPRS Geospatial Week 2019, 10-14 June 2019, Enschede, The Netherlands. https://www.int-arch-photogrammremote-sens-spatial-inf-sci.net/XLII-2-W13/343/2019/isprsarchives-XLII-2-W13-343-2019.pdf (11 June 2021).

Hanus, P., Pęska-Siwik, A., Szewczyk, R., 2017: Spatial analysis of the accuracy of the cadastral parcel boundaries. Computers and Electronics in Agriculture. Volume 144, January 2018, Pages 9-15. https://doi.org/10.1016/j.compag.2017.11.031.

He, G. B., Li, L. L., 2020: Research and application of LiDAR technology in cadastral surveying and mapping. The International Archives of the Photogrammetry, Remote Sensing and Spatial Information Sciences, Volume XLIII-B1-2020, 2020 XXIV ISPRS Congress (2020 edition). https://www.intarch-photogramm-remote-sens-spatial-inf-sci.net/XLIII-B12020/33/2020/isprs-archives-XLIII-B1-2020-33-2020.pdf (11 June 2021)

Kedzierski, M., Fryskowska, A., Wierzbicki D., Nerc, P., 2016: Chosen aspects of the production of the basic map using UAV imagery. The International Archives of the Photogrammetry, Remote Sensing and Spatial Information Sciences, Volume XLI-B1, 2016 XXIII ISPRS Congress, 12-19 July 2016, Prague, Czech Republic. https://www.int-arch-photogrammremote-sens-spatial-inf-sci.net/XLI-B1/873/2016/isprs-archivesXLI-B1-873-2016.pdf (11 June 2021).

Kurczynski, Z., Bakuła, K., Karabin, M., Kowalczyka, M., Markiewicz, J. S., Ostrowski, W., Podlasiak, P., Zawieska, D., 2016: The possibility of using images obtained from the UAS in cadastral works. The International Archives of the Photogrammetry, Remote Sensing and Spatial Information Sciences, Volume XLI-B1, 2016 XXIII ISPRS Congress, 1219 July 2016, Prague, Czech Republic. https://www.int-archphotogramm-remote-sens-spatial-inf-sci.net/XLI-

B1/909/2016/isprs-archives-XLI-B1-909-2016.pdf (11 June 2021).

OpenDroneMap Develpoment Team, 2020: A command line toolkit to generate maps, point clouds, 3D models and DEMs from drone, balloon or kite images. OpenDroneMap/ODM GitHub Page 2020. https://github.com/OpenDroneMap/ODM (11 June 2021).

Murtiyoso, A., 2021: M_HERACLES. HERitAge by point CLoud procESsing for Matlab.

https://github.com/murtiad/M_HERACLES (11 June 2021).

Neteler, M., Mitasova, H., 2008: OpenSource GIS: A GRASS GIS Approach - $\quad 3^{\text {rd }}$ edition Springer, ISBN-10: 038735767X, ISBN-13:978-0387357676. Nguyen, A., Le, B., 2013: 3D Point Cloud Segmentation: A survey. Conference: 2013 6th International Conference on Robotics, Automation and Mechatronics (RAM) (2013). https://www.researchgate.net/publication/269035150_3D_point _cloud_segmentation_A_survey (11 June 2021). 
Rijsdijk, M., van Hinsbergh, W.H.M., Witteveen, W., ten Buuren, G.H.M., Schakelaar, G.A., Poppinga, G., van Persie, M., Ladiges, R., 2013: Unmanned arial systems in the process of juridical verification of cadastral border. International Archives of the Photogrammetry, Remote Sensing and Spatial Information Sciences, Volume XL-1/W2, 2013 UAV-g2013, 4 - 6 September 2013, Rostock, Germany. https://www.int-archphotogramm-remote-sens-spatial-inf-sci.net/XL-1-

W2/325/2013/isprsarchives-XL-1-W2-325-2013.pdf (11 June 2021).

Taylor, Z., 2021: Find 3D Normals and Curvature. MATLAB Central File Exchange. Retrieved March 25, 2021. https://uk.mathworks.com/matlabcentral/fileexchange/48111find-3d-normals-and-curvature (11 June 2021).

Zhang, W., Qi, J., Wan, P., Wang, H., Xie, D., Wang, X., Yan, G., 2016: An Easy-to-Use Airborne LiDAR Data Filtering Method Based on Cloth Simulation. Remote Sensing. 2016; 8(6):501.

https://www.researchgate.net/publication/303975654_An_Easyto-

Use_Airborne_LiDAR_Data_Filtering_Method_Based_on_Clot h_Simulation (11 June 2021).

Zhou, Q.Y., Park, J., Koltun V., 2018: Open3D: A Modern Library for 3D Data Processing. arXiv: 1801.09847. https://arxiv.org/pdf/1801.09847.pdf (11 June 2021). 